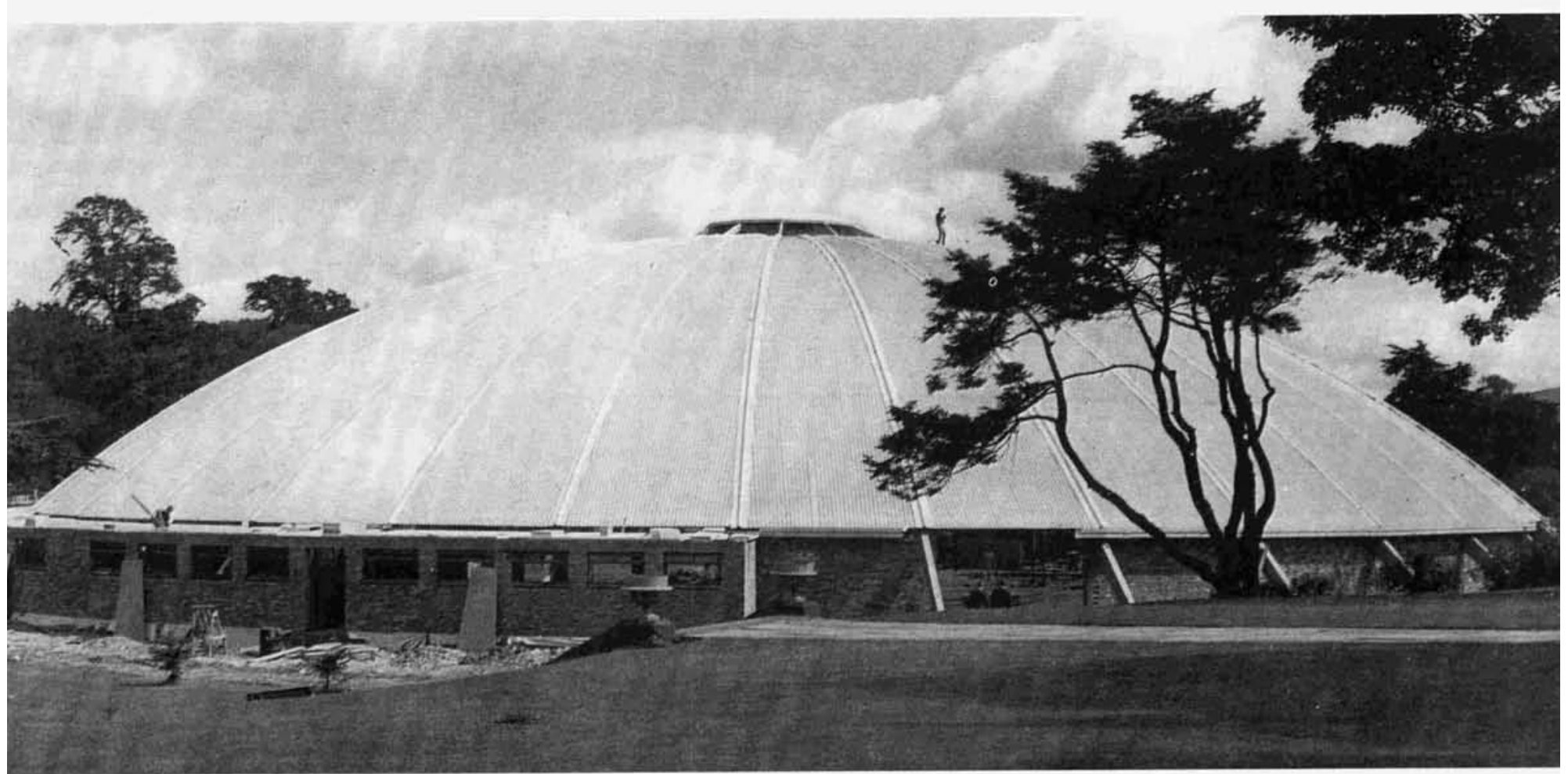

\title{
centro de deportes BELL en Perth - Gran Bretaña
}

JOHN B. DAVIDSON, T. R. WALKER y J. H. CARNEGIE, arquitectos

simopesis

$158-4$

Tras una serie de minuciosas consideraciones de tipo económico y estático se llegó a la solución de cubrir el edificio con una cúpula, cuyos elementos resistentes se realizaron con madera laminada. Dicha cúpula está constituida por 18 nervios completos, unidos en su parte superior mediante un anillo central, y en su parte inferior con una viga-tirante de hormigón. Los nervios, que tienen $35,96 \mathrm{~m}$ de longitud, fueron construidos a base de tablas de madera del Báltico, arriostrados transversalmente con barras de acero dulce, las cuales contribuyen, junto con las correas concéntricas colocadas a una distancia de $1,60 \mathrm{~m}$ entre ejes, a la estabilidad de la cúpula.

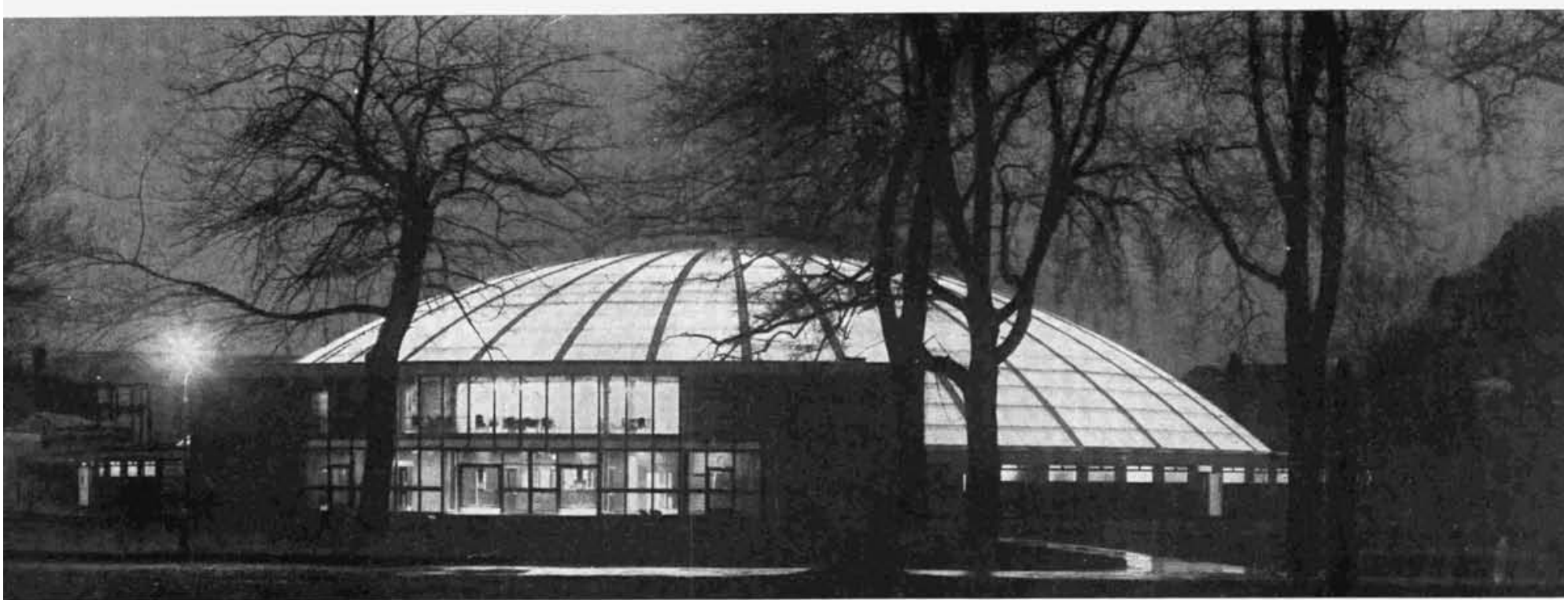


Fue construido - aprovechando el declive de North Inch- con el fin de fomentar, en el centro de la ciudad de Perth, las actividades deportivas, satisfaciendo un programa de necesidades en el que figuraban: la construcción de una gran sala de $36,58 \times 36,58 \mathrm{~m}$, y las de entrenamiento, juegos interiores, etc.

Las obras se realizaron, a pesar de las inclemencias meteorológicas, en el plazo récord de diez semanas.

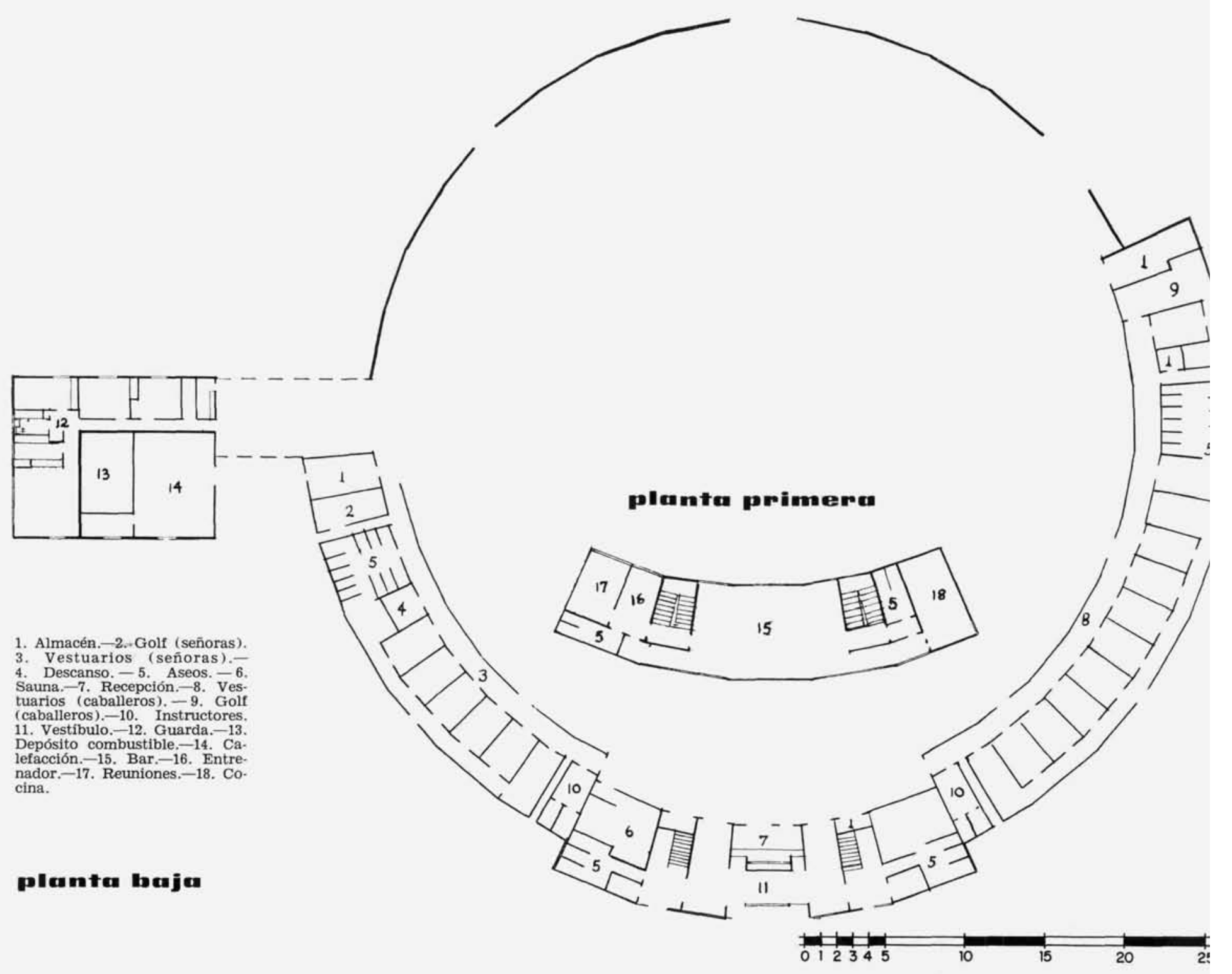

\section{sección A-A}

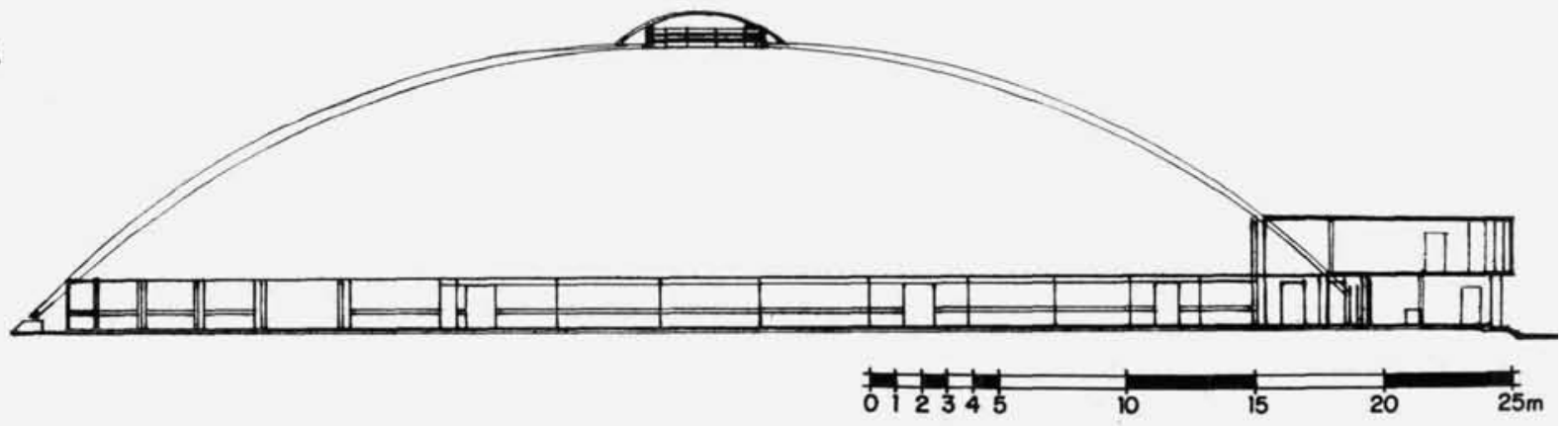




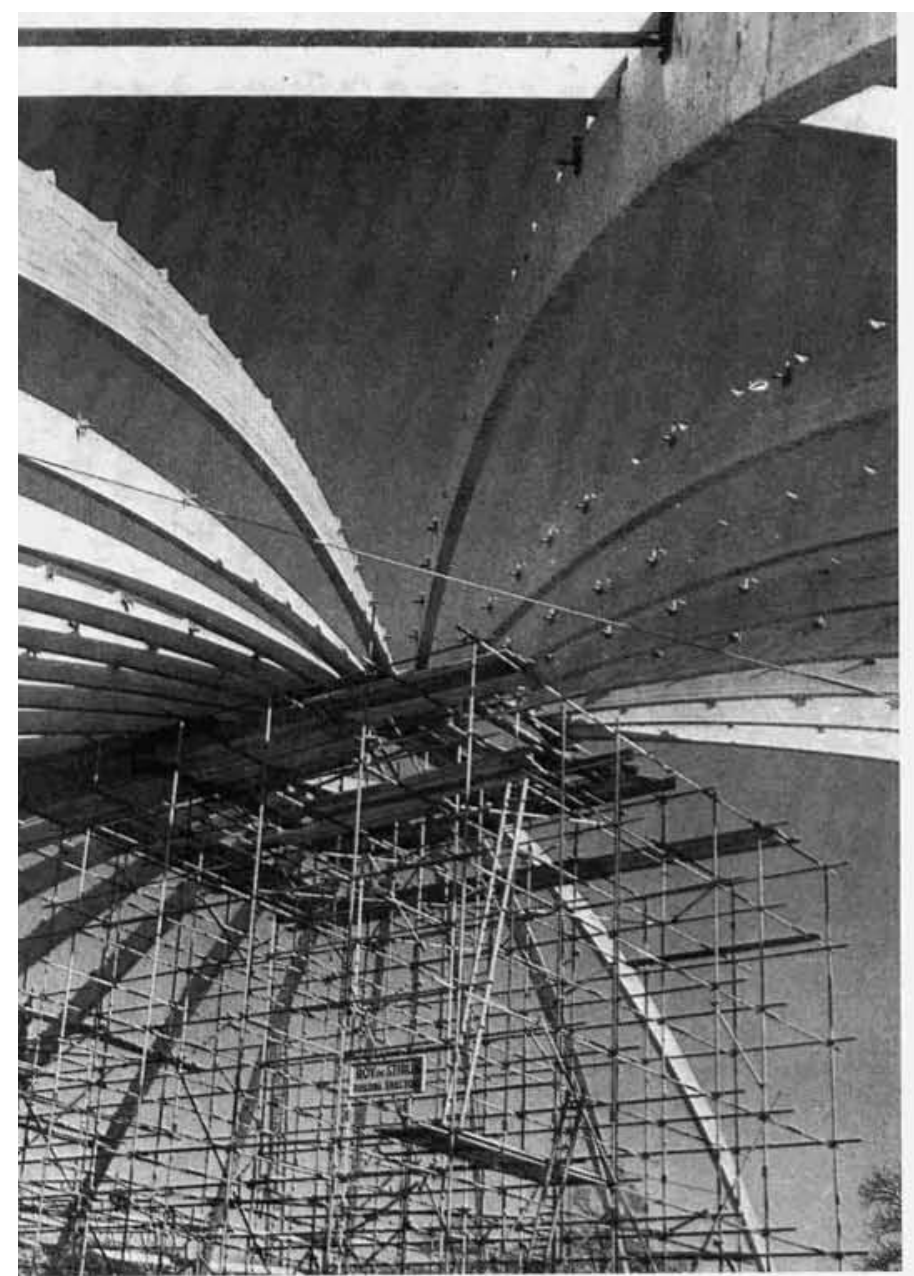

En la fase de proyecto y tras un minucioso estudio de los costos de diferentes tipos de estructuras - metálica, de hormigón y de madera laminaday de otra serie de consideraciones de orden financiero y estético, el arquitecto se decidió por una estructura con forma de cúpula realizada a base de madera laminada.

La "forma" de los elementos de la estructura de la cubierta, diseñada pensando en que fuese lo más económica posible, es fundamentalmente la de una catenaria, estando constituida dicha estructura por una serie de nervios que se extienden radialmente de dentro afuera.

En la construcción de la cúpula, formada por 18 nervios completos, resultaron delicadas y peligrosas: la operación de enlace de dichos nervios en la cima de la cúpula, y la de unión de los nervios con el tirante; para ello, fueron construidos: en la parte superior de la cúpula, un anillo central para absorción de empujes y llevadas a cabo 36 uniones con acero reforzado para altas tensiones; y en la parte inferior, vigas-tirante de hormigón moldeado in situ; las extremidades de los nervios son de acero y forman una especie de caja metálica.

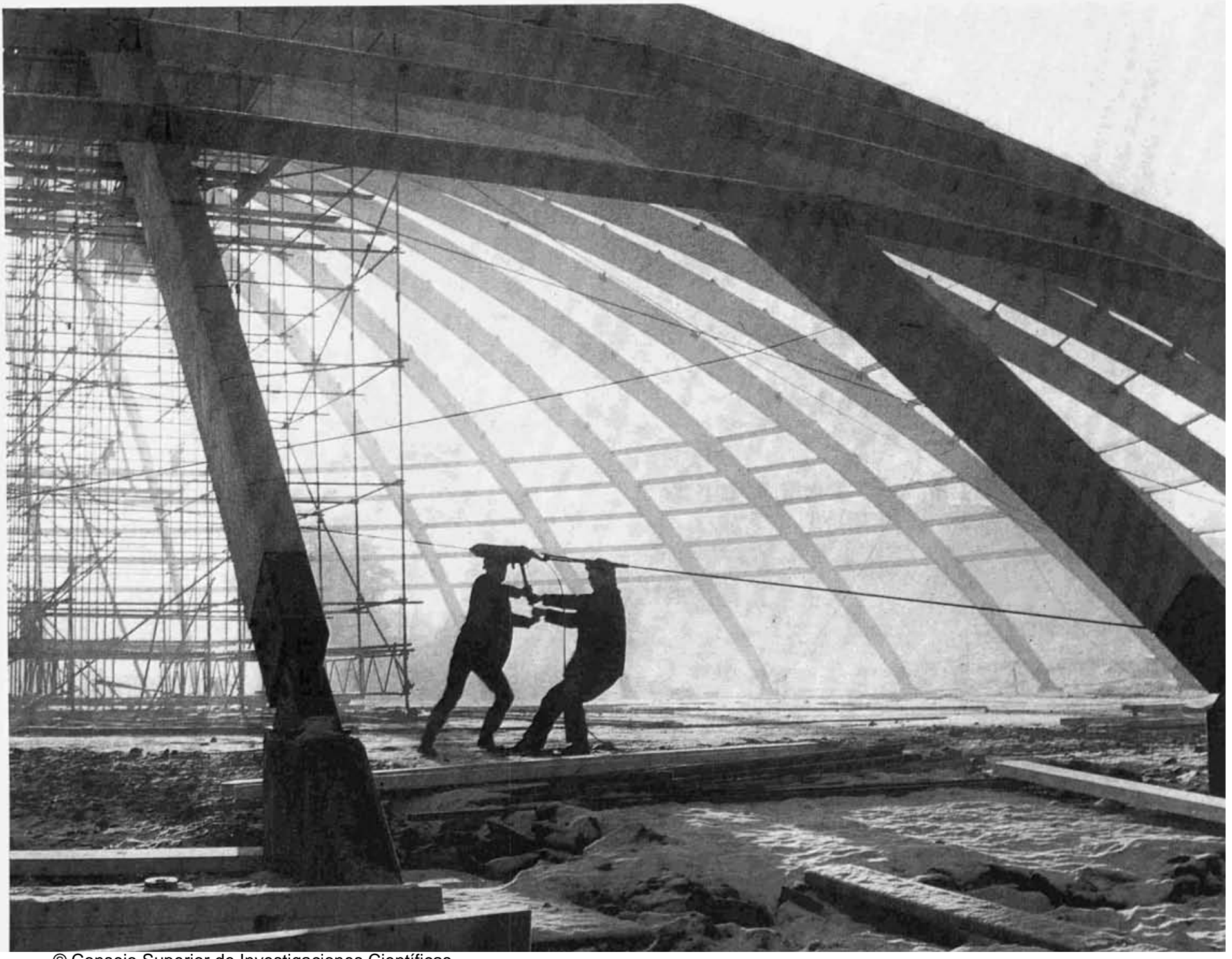



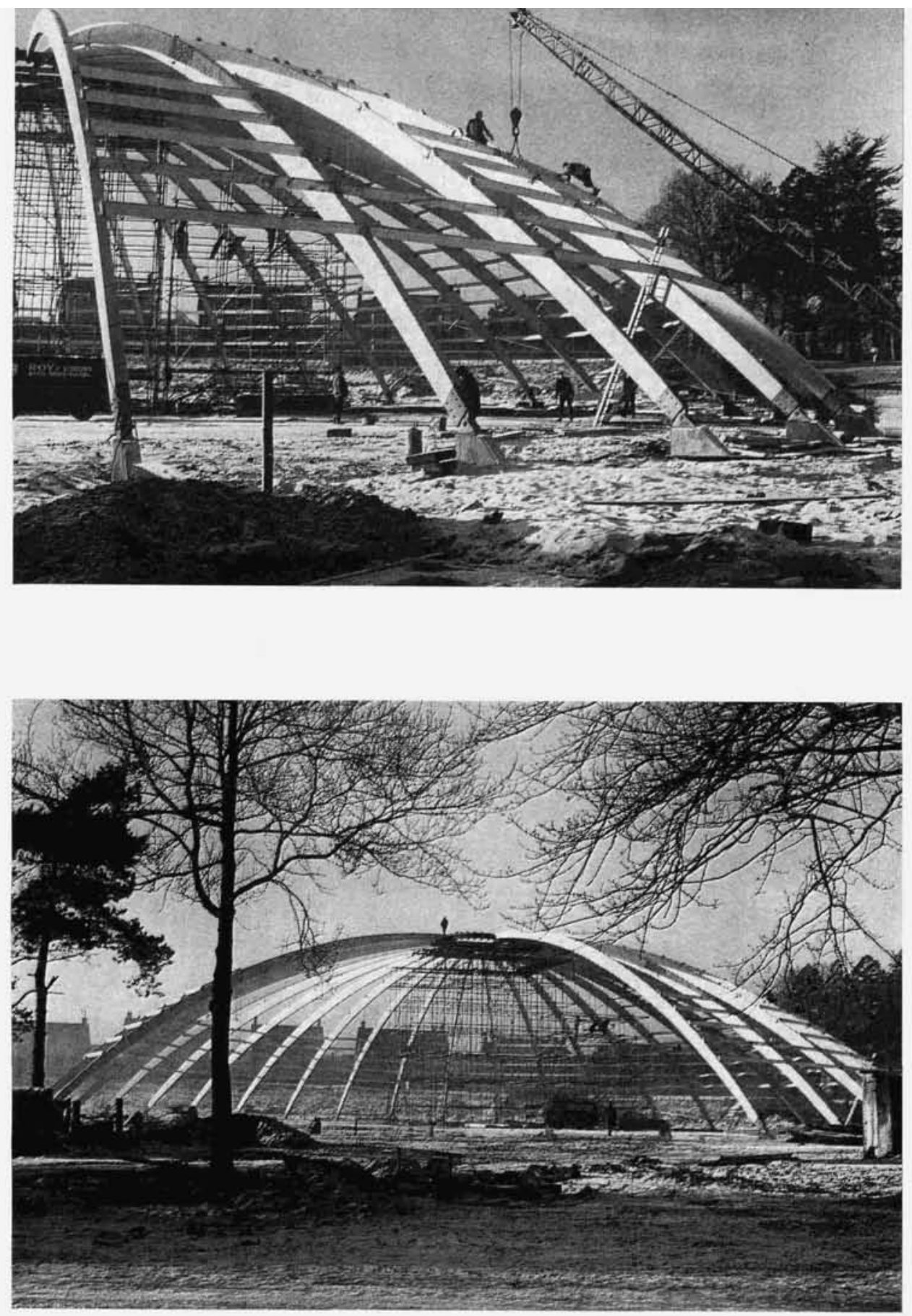

Los nervios, que tienen 35,96 m de longitud, están formados por tablas de madera del Báltico, de $4,7 \mathrm{~cm}$ de grueso cada una, unidas, por tramos de $30,48 \mathrm{~cm}$, con un pegamento de resorcinol, comprimiéndolas sobre guías prefabricadas.

La forma de los nervios obedece a un perfil de 46,94 metros de radio exterior de curvatura y $48,77 \mathrm{~m}$ de radio interior; tales radios, variables, proporcionaron una base de $45,7 \mathrm{~cm}$; otra, de $38,1 \mathrm{~cm}$, en la parte superior, y $76,2 \mathrm{~cm}$ en el punto de momento flector máximo.

La estabilidad de la cúpula se consiguió mediante el arriostramiento de los nervios, realizado con ayuda de barras de acero dulce de $3 / 4$ " como riostras transversales -bulonadas a la base en cada $1 / 3$ del vano-, y las correas concéntricas colocadas a lo largo de las líneas de curvatura y a una distancia entre ejes de 1,60 m; estas correas presentan un revestimiento de color azul, con fines estéticos, además de ofrecer una transmisión luminosa del $62 \%$, ser antideslumbrantes, y tener la particularidad de absorber los rayos ultravioletas.

El alumbrado artificial ha sido resuelto mediante anillos luminosos concéntricos de luz fluorescente, y de una serie de reflectores regulables montados en la parte superior del anillo de empujes. El sistema de interruptores permite, en el alumbrado, la posibilidad de poder conseguir 336 combinaciones distintas.

Revestimientos de suelos. El pavimento ha sido construido a base de Gramwood, material que contiene un conglomerante de serrín, oxidado e impregnado con petróleo, formando unas losetas que se reciben

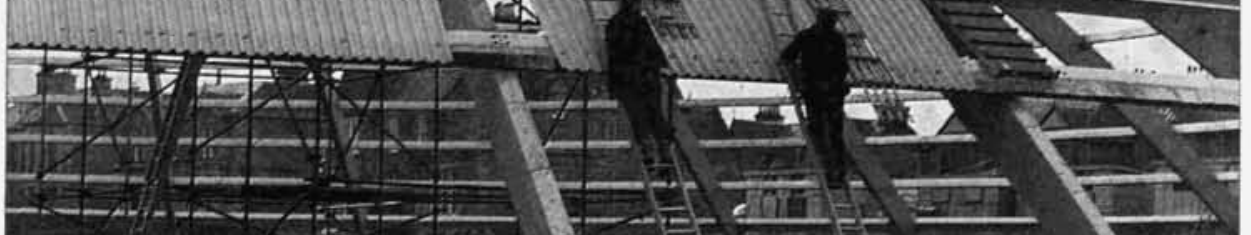


con una mezcla de cemento y arena. Este tipo de solado fue elegido por ser el más adecuado para el rebote del balón y para facilitar las carreras pedestres, tras consultar a médicos, entrenadores y atletas.

Finalmente, señalaremos que el bloque de dos plantas de uso público fue construido, asimismo, con una estructura entramada de madera laminada, que acreditó de nuevo ser la forma estructural considerablemente más económica.

\section{Bentre de sponts Bell ì Perth - Grande-Bretrgine}

John B. Davidson, T. R. Walker et J. H. Carnegie, architectes

Après une série de considérations minutieuses de type économique et statique, il fut décidé de couvrir l'édifice d'une coupole dont les éléments résistants ont été réalisés en bois lamellé.

Cette coupole est constituée par 18 nervures complètes, reliées à leur extrémité supérieure à un anneau central et, à leur extrémité inférieure, à une poutre-tirant en béton.

Les nervures, de $35,96 \mathrm{~m}$ de long, ont été réalisées en planches de bois de la Baltique, raidies transversalement à l'aide de barres en acier doux qui, avec les pannes concentriques placées à une distance de $1,60 \mathrm{~m}$ entre axes, contribuent à la stabilité de la coupole.

\section{Bell Sponts Eantme, in Perth - At. Britaim}

John B. Davidson, T, R. Walker and J. H. Carnegie, architects

After detailed research on the statics and cost of the project, it was decided to roof over the building with a dome, whose structural part consists of laminated timber.

This dome has 18 full ribs, joined at the top by a circular member and at the bottom by a concrete tie beam.

The ribs are each $36 \mathrm{~m}$ long, and were made with Baltic timber boards, and are transversally stabilised with mild steel bars. In this function the bars are aided by concentric joists, distanced $1.60 \mathrm{~m}$ from each other.

\section{Sportzentrume Bell in Perth - Gross Britammien}

John B. Davidson, T. R. Walker und J. H. Carnegie, Architekten

Nach eingehenden wirtschaftlichen und ästhetischen überlegungen entschloss man sich zu der Lösung, das Gebäude mit einer Kuppel zu bedecken, deren Stützelemente aus Holzträgern bestehen.

Diese Kuppel setzt sich aus achtzehn Rippen zusammen, die am oberen Ende durch einen zentralen Ring und am unteren Ende durch einen Bindepfeiler aus Beton verbunden sind.

Die Rippen haben eine Länge von $35,96 \mathrm{~m}$ und wurden aus Holklatten aus dem Baltikum hergestellt, die mit Querbeschlägen aus Süsstahl versehen wurden und die ebenso wie die konzentrischen Glieder in einer Entfernung von 1,60 m zwischen Achsen zur Stabilität der Kuppel beitragen. 